\title{
Coronal Mass Ejections, Solar Cycles and Magnetic Poles Reversal
}

\author{
Kim Kwee Ng \\ Department of Physics and Astronomy, State University of New York at Stony Brook, New York, USA \\ Email address: \\ ng@sbhep.physics.sunysb.edu \\ To cite this article: \\ Kim Kwee Ng. Coronal Mass Ejections, Solar Cycles and Magnetic Poles Reversal. American Journal of Astronomy and Astrophysics. \\ Vol. 7, No. 1, 2019, pp. 10-17. doi: 10.11648/j.ajaa.20190701.12
}

Received: June 19, 2019; Accepted: July 12, 2019; Published: July 26, 2019

\begin{abstract}
The magnitude of the measured geomagnetic index increases when the Coronal Mass Ejections occur on the Sun's surface. The abrupt increase in the geomagnetic index has seriously impacted the accuracy in the forecast of the activity of the next solar cycle. A method is proposed to filter the effect from the Coronal Mass Ejections. The correlation between the geomagnetic index and the activity of the subsequent solar cycle is found to have drastically improved with the proposed scheme. A strong correlation between the maximum amplitude $\mathrm{R}_{\mathrm{N}}$ of a solar cycle $\mathrm{N}$ and its pre-cycle coronal mass ejections adjusted monthly geomagnetic activity index has been qualitatively determined, as illustrated by an impressive correlation coefficient of $0.91+0.09-0.12$, with its statistical significance estimated at $4.3 \sigma$. The corrected data have significantly improved the correlation between the observed variables from their original un-corrected case of $0.63 \pm 0.23$. Our result indicates that the upcoming solar cycle, estimated at $\mathrm{R}_{25}=147 \pm 30$, would be stronger than the current waning solar cycle 24 . In a related calculation, the magnetic poles reversals occurring in the solar cycles 21 and 22 are reproduced numerically from Maxwell's electromagnetic equations.
\end{abstract}

Keywords: Sunspots, Solar Flares, Solar Cycles, Precursor Method, Geomagnetic Activity, Magnetic Field, Magnetic Poles Reversal

\section{Introduction}

The activity of the Sun has long been a very important topic. Solar events include solar flares, coronal mass ejections (CMEs), magnetic poles reversal and their associated solar cycles. Motivations for better understanding these phenomena include the fact that high-energy charged particles and short-wavelength photons produced from the coronal mass ejections are dangerous to satellites and astronauts, and have been known to cause wide spread communication interruption and equipment failures. For example, a massive power blackout in Quebec, Canada was caused by a large CME in March 1989 [1].

Coronal mass ejections are one of the dominant ways that solar activity could affect the accuracy in the prediction of future solar activity, when the geomagnetic index and other similar data are employed in the analysis of the solar activity. A new scheme is proposed below to compensate for the elevated values caused by the coronal mass ejections (CMEs). The correlation between the geomagnetic index and the maximum amplitude $\mathrm{R}_{\mathrm{N}}$ of the subsequent solar cycle $\mathrm{N}$ is found to have drastically improved with the corrected data.

As the current solar cycle 24 comes to a close, a new cycle with a maximum amplitude $\mathrm{R}_{25}$ weaker than the previous few solar cycles has been predicted [2, 3]. This would continue the decline of the solar activity over the last few solar cycles. The maximum 13-month smoothed sunspot number in the last solar cycle was $\mathrm{R}_{24}=116.4$ in April 2014, it was only about $40 \%$ of the peak recorded in 1958 . The $13-$ month smoothed sunspot number is a running mean of the monthly sunspot numbers over 13 months centered on the corresponding month, with a one-half of the weight assigned to the first and the last months in the series. The solar activity peak, $\mathrm{R}_{\mathrm{N}}$, found in the 13-month smoothed sunspot numbers over the full cycle of the solar cycle having a designated number $\mathrm{N}$, is often considered a representative of the strength of the solar cycle $\mathrm{N}$.

Many approaches have been attempted to predict the activity of the next incoming solar cycle. Their methods and the results have been summarized by Pesnell [4]. For a much 
longer term prediction, a dip in the solar activities beyond 2030 has been forecast [2]. As to the forthcoming solar cycle 25, Gkana and Zachilas have predicted $R_{z}=54.4$ [3] (where $R_{z}$ is Version 1 of the International Sunspot Number and is equivalent to $R_{25} \sim 80$ ), and $R_{z}=50$ from Javaraia [5].

In contrast to the above results, some earlier works using different methods have pointed toward a higher solar activity. Miao et al. obtain $R_{25} \sim 185$, or $R_{z}=119.2 \pm 5.5$ [6]. The result from Helal and Galal [7] is $R_{z}=118.2$, and from Yoshida [8] $\mathrm{R}_{\mathrm{z}}=115.4 \pm 11.9$. Lampropoulos et al. get $\mathrm{R}_{\mathrm{z}}=$ $157 \pm 33$ [9]. A recent study, which uses solar polar magnetic field and solar spectral irradiance at $10.7 \mathrm{~cm}$, has predicted $\mathrm{R}_{25}=135 \pm 25$ [10]. The predicted number is quite close to the results presented in this work.

The correlation between the geomagnetic activity index and the subsequent solar activity has been explored in the past [11-14]. The current efforts are traceable to some earlier works done by Ohl [15], Thompson [16] and Feynman [17].

The corrected data produced from the proposed scheme are described below. We describe here how recent corrected geomagnetic data, measured in terms of the activity index aa, though still limited, point to a new solar cycle 25 that will be stronger, statistically speaking, than the current solar cycle 24. Moreover, an analysis has been made on the observed Sun's magnetic poles reversals which could have occurred under different initial conditions for the solar cycles 21 and 22.

\section{Methods}

\subsection{Observation of Data}

The geomagnetic activity index aa is derived from the $\mathrm{K}$ indices from two nearly antipodal observatories on Earth [18]. A $\mathrm{K}$ index is a quasi-logarithmic local index of the magnetic activity measured in 3-hourly periods, relative to an assumed quiet day for each geomagnetic observatory site. The activity index aa is thus a measure of the disturbances in the Earth's magnetic field, most likely coming from solar activity, including coronal mass ejections.

Coronal mass ejections (CME) striking the Earth elevate the activity index locally in time, thus making the forecast of the solar activity for a new solar cycle less reliable. Other coronal mass ejections have since been studied after they were detected in the last few decades. One example was the Halloween solar storms which occurred in October of 2003. However, the historical data on the strength and occurrences of coronal mass ejections only cover the last 30 years. The number of recorded events is insufficient for the data analysis. It has been observed that the coronal mass ejections are often produced after the eruption of solar flares. Thus, the collected geomagnetic index aa could be approximately revised when a simplified scheme to remove the impact from the coronal mass ejections is adopted as shown below.

\subsection{Method of Calculation}

A new scheme for the modified use of the observed geomagnetic activity index is now described in detail below.

The observed daily measured geomagnetic activity index, which reflects the sunspots activity and the magnetic disturbances occurring in the Sun, is scanned for exceptionally high values $\mathrm{aa}_{\max }$ of data, e.g. $\mathrm{aa}_{\max }=30$. When aa $>\mathrm{aa}_{\max }$, the sunspot number for that day and the previous two days must each exceed a threshold value, $\mathrm{N}_{\text {sun }}=$ 40. During a typical quiet day when there is no observable solar flare, the daily geomagnetic activity index aa is observed to vary between $0-30$, the daily sunspot number is about $0-30$. When the above conditions are met, a new CME-adjusted daily geomagnetic activity index aa for that day is calculated as the mean of the previous five daily aa values, as shown in Equation (1),

$$
\overline{a a}(\text { day })=\frac{1}{5} \sum_{\text {day }=-1}^{-5} a a(\text { day })
$$

The CME-adjusted geomagnetic activity index aa calculated for the day is applied when an exceptionally high value of geomagnetic activity index aa is found on the following day of continuing strong activity. The geomagnetic activity index is adjusted to remove the effect of the coronal mass ejections and to improve the forecast of the solar activity in the next solar cycle.

In summary, the daily geomagnetic activity index is scanned for signs of elevated activity. It has been known that there is a time delay for the charged particles from the CME to reach the Earth. However, an unusual geomagnetic activity would be recorded only after the CME has reached the Earth to cause the magnetic disturbances in the Earth's atmosphere.

\section{Results}

The solar activity peak, $R_{N}$, found in each solar cycle and its counter-part, the peak found in the pre-cycle CMEadjusted monthly geomagnetic activity index aa are plotted in Figure 1. Figure 1 shows an interesting picture in which the curves are seen to be in a synchronized motion, despite the fact the geomagnetic index and the observed sunspot numbers are two distinct physical parameters determined at different places. The peak found in the adjusted monthly geomagnetic activity index aa is chosen about 2 years prior to the effective start of the solar cycle. A peak which appears first in the daily average of the sunspot numbers in each solar cycle is also plotted. 


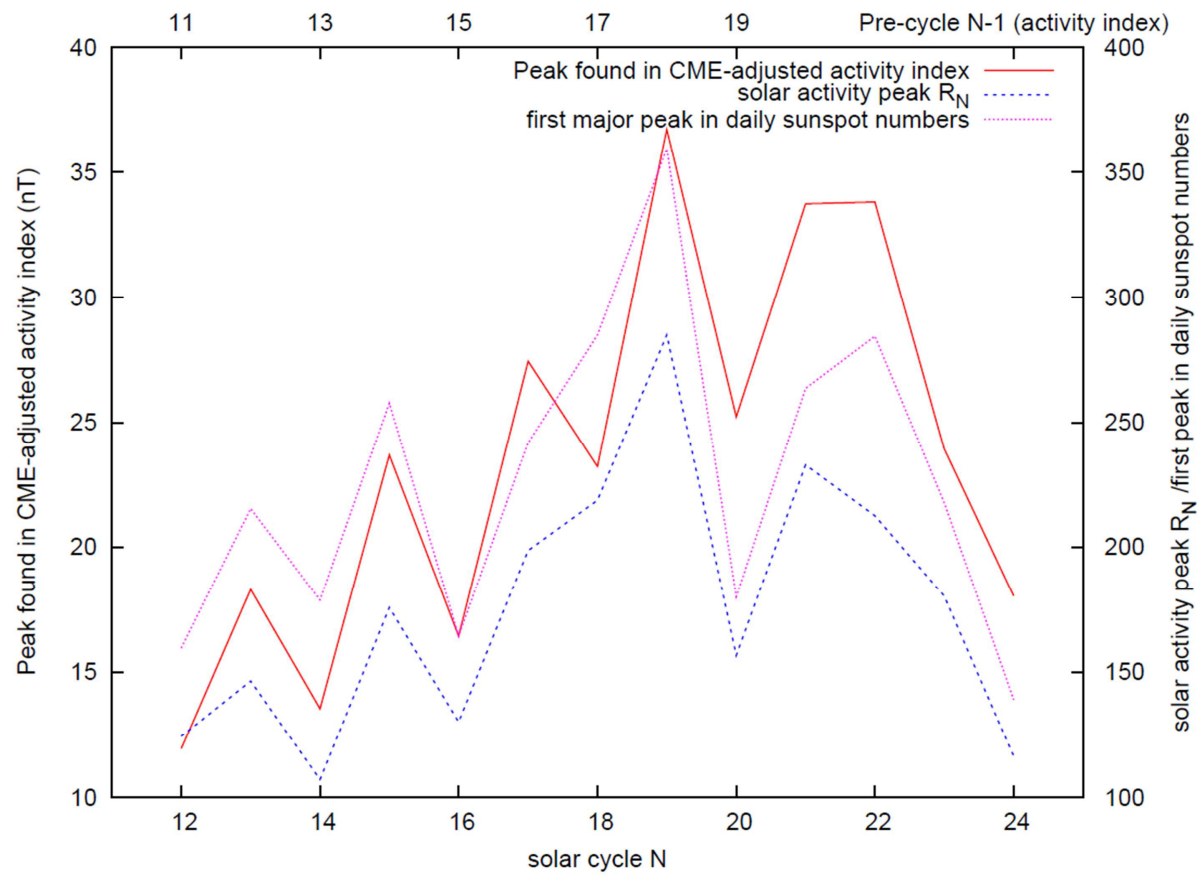

Figure 1. Pre-cycle peak found in the CME-adjusted activity index, solar activity peak $R_{N}$, and the first major peak in daily averaged sunspot numbers in solar cycle $N$.

Figure 2 shows the linear relationship between the solar activity peak $R_{N}$ found in the solar cycle $\mathrm{N}$ and the peak found in the pre-cycle, N-1, CME-adjusted monthly geomagnetic activity index aa. The Pearson's correlation coefficient is calculated to be $\rho$ (Pearson $)=0.91+0.09-0.12$, which implies that the correlation between the paired variables is very strong.

To get an estimate of the confidence level of the result, a T-test, which usually applies to a normal distribution, is performed. The t-value is $t=7.33, d f=11$, and $\mathrm{p}$-value is 7.4 $\times 10^{-6}$ (right tail). The statistical significance is determined to be $4.3 \sigma$. The result calculated by a different method called Spearman method, which assigns different ranks to the data that may be distributed like a non-Gaussian distribution, yields a value of $\rho$ (Spearman $)=0.92+0.08-0.12$. The $\mathrm{t}-$ value is $t=7.65, d f=11$, and P-value is $5.0 \times 10^{-6}$ (one-tail). The statistical significance is estimated to be $4.4 \sigma$. Without the corrective adjustment that removes the elevated effects from the coronal mass ejections described above, the Pearson's correlation factor in the un-corrected case is $\rho($ Pearson $)=0.63 \pm 0.23, t=2.70$. The $\mathrm{p}$-value is 0.01 , which implies a confidence level of $2.3 \sigma$.

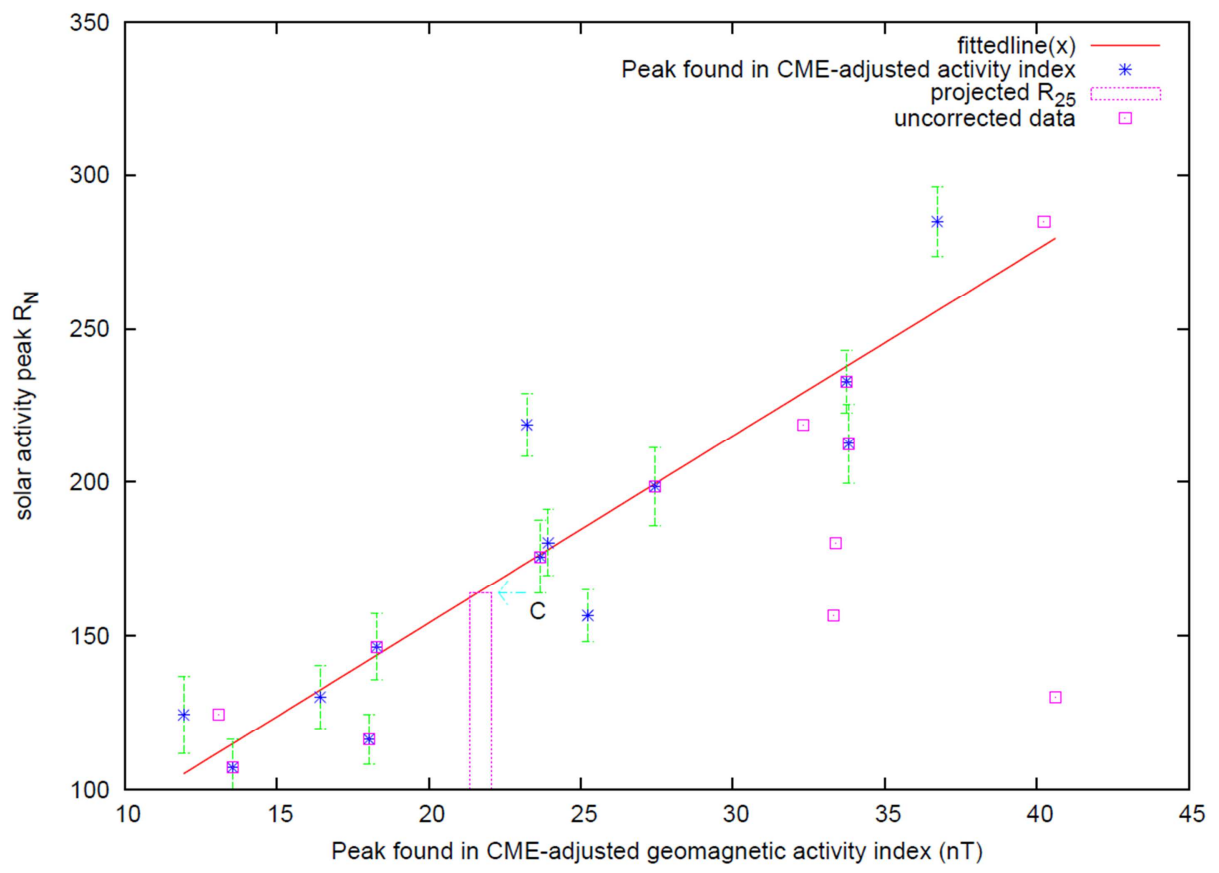

Figure 2. Pre-cycle peak found in the CME-adjusted activity index and solar activity peak $R_{N}$, in the subsequent solar cycle $N$. 


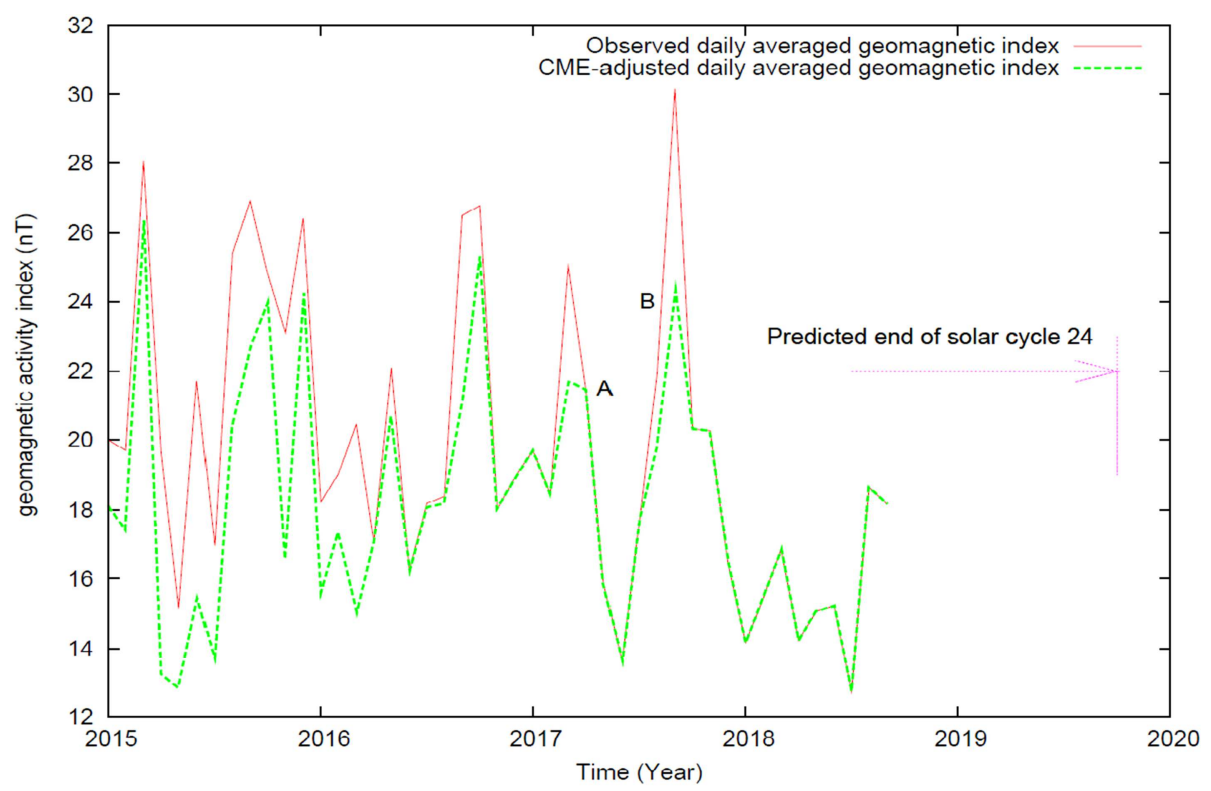

Figure 3. Pre-cycle peak found in the CME-adjusted activity index before the start of solar cycle 25.

The new solar cycle 25 was independently predicted to begin in late 2019 [19]. It may be useful to look for changes in the observed geomagnetic activity index two years in advance. A pair of precursor peaks, "A" and "B" which are found near the two-years selection criteria prior to the start of the upcoming solar cycle 25, is shown in Figure 3. The precursor peaks are found to have produced high correlation factors in the study. The first precursor peak " $\mathrm{A}$ " is registered in March 2017 in the recently collected geomagnetic activity index aa. The corresponding predicted maximum 13-month smoothed sunspot number in the upcoming solar cycle 25 from the first precursor peak "A" is estimated to have a value of $R_{25}=164 \pm 30$, which is labeled as " $\mathrm{C}$ " in Figure 2. The currently collected CME-adjusted accumulated daily activity index aa, starting from April 2017, has implied a value, $R_{25}=$
$130 \pm 30$. Current estimate is the combination of the two results above, at $R_{25}=147 \pm 30$. The solar storms are a major factor in the uncertainty of the prediction on the expected amplitude $R$ of the upcoming solar cycle. More study on the solar storms would help to further improve the accuracy in the forecast of the solar activity.

Figure 4 shows a good correlation between the pre-cycle peak found in the CME-adjusted monthly geomagnetic activity index aa and the peak which appears first in the daily average of the sunspot numbers in each solar cycle. The correlation is calculated to be $\rho$ (Pearson $)=0.83 \pm 0.17$, the statistical significance is estimated at $3.5 \sigma$. The current projection for the first peak in the daily average of sunspot numbers in the upcoming solar cycle is $214 \pm 40$ (" $\mathrm{D}$ " in Figure 4).

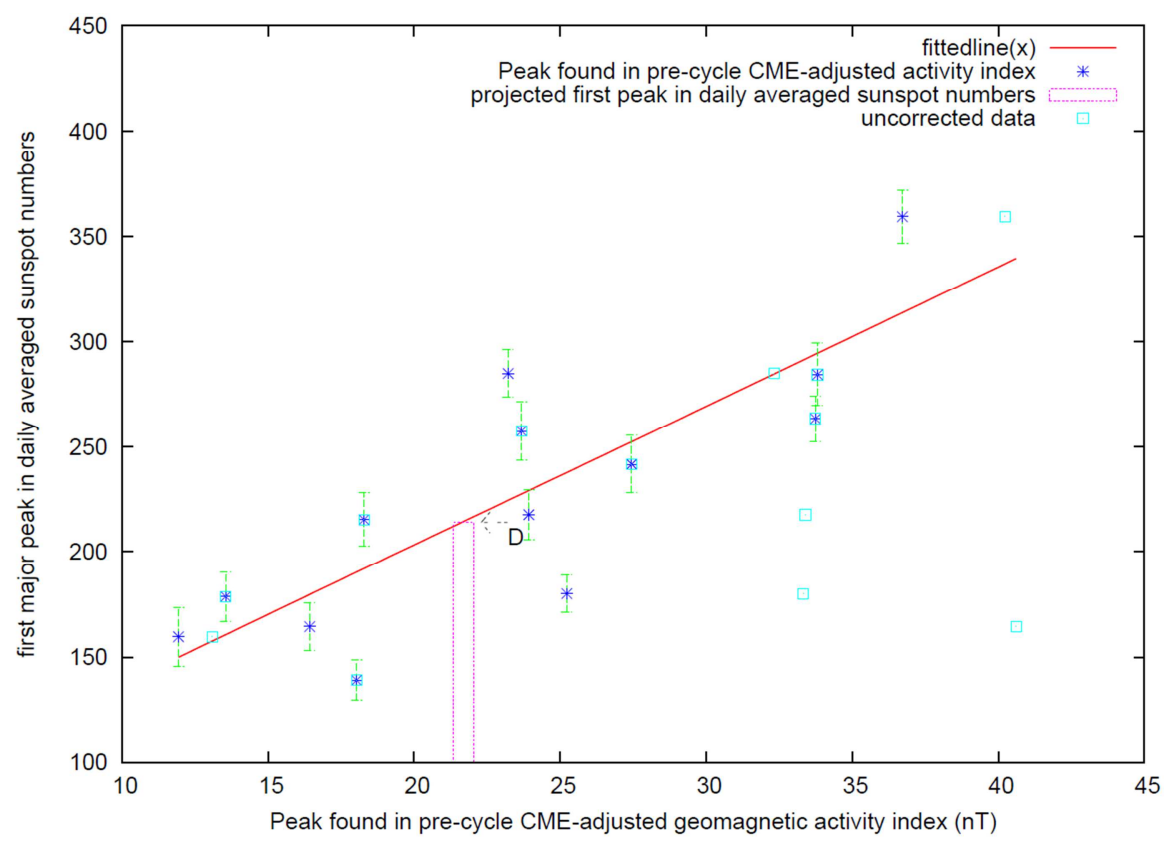

Figure 4. Pre-cycle peak found in the CME-adjusted activity index and the first major peak in daily averaged sunspot numbers in the subsequent solar cycle. 


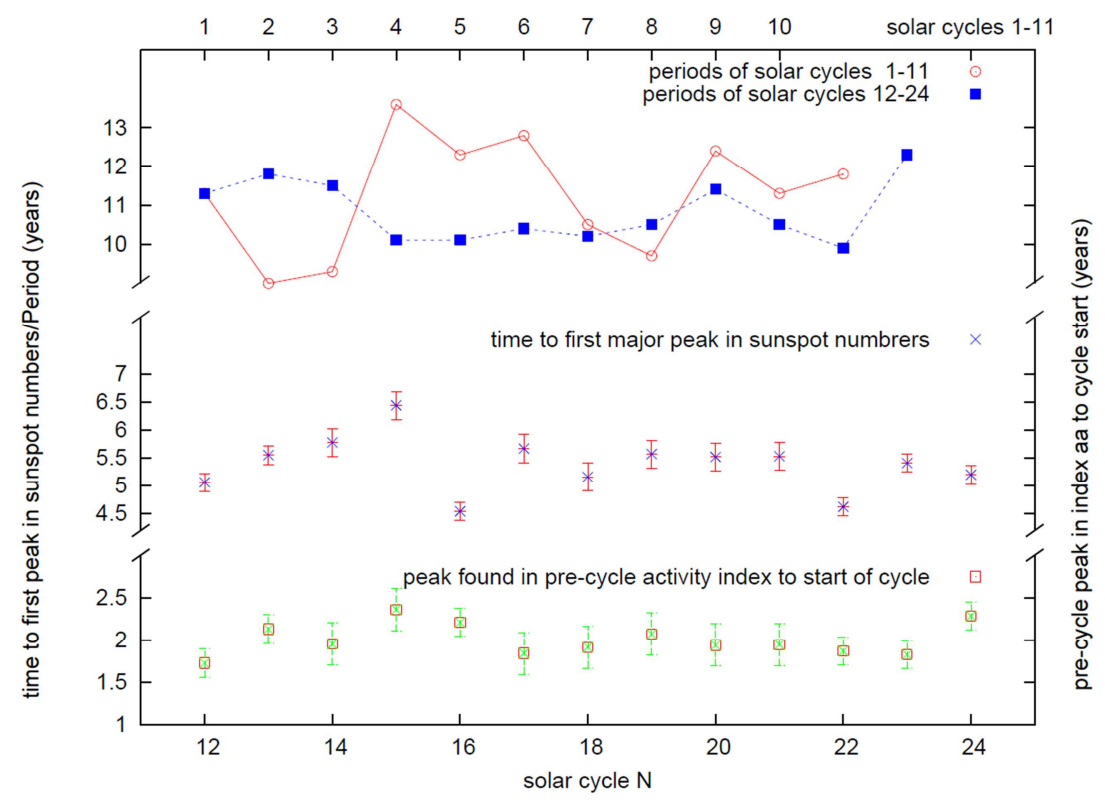

Figure 5. Time differences between pre-cycle peak found in CME-adjusted activity index and other solar events in the subsequent solar cycle.

The strength of the adjusted aa index is found to be strongly correlated to the maximum amplitude $R_{N}$ of the subsequent solar cycle $\mathrm{N}$ as illustrated in Figure 2. The evidences shown by the strong correlation described above have demonstrated that the variation in the Sun's polar magnetic field is strongly correlated to the solar activity in the subsequent solar cycle. The change in the magnetic field arising from the Sun's activity is recorded by the magnetometers on the Earth. The data are translated into the observed geomagnetic activity index used in this study.

The arrival time of the next solar cycle is characterized by the first appearance of a positive or negative polarity-leading sunspot in the high latitude, opposite in magnetic polarity to the previous solar cycle. However, the arrival time is different from the effective start time of a solar cycle which is usually assumed to be solar minimum. As shown in Figure 5 , the average time to go from a pre-cycle peak found in the adjusted aa index to solar minimum (the start of the next solar cycle) is $2.1 \pm 0.5$ years when the solar minimum occurs. Based on the current data, as shown by the peak " $A$ " in Figure 3 and the averages calculated from Figure 5, it is expected that the new solar cycle 25 would start in May 2019 \pm 6 months. The second peak " $B$ " shown in Figure 3 may imply a delay start of about 6 months. Figure 5 also shows the periods of the past solar cycles 1-23. The average time difference between the pre-cycle peak found in the geomagnetic index aa and the first major peak in the daily average of the sunspot numbers in the subsequent solar cycle is also plotted. The mean is $5.4 \pm 1.2$ years.

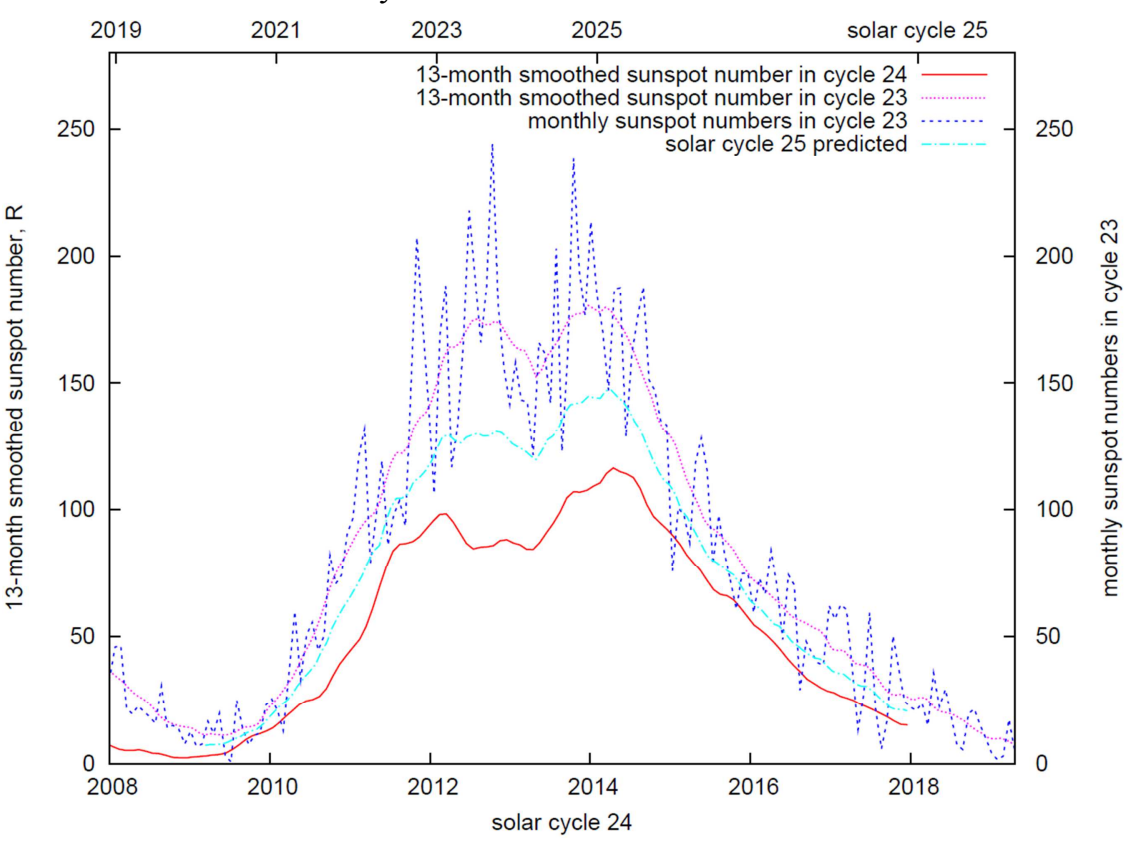

Figure 6. Solar cycles 23, 24 and the upcoming solar cycle 25. 
The 13-month smoothed sunspot numbers in the current solar cycle 24 are shown in Figure 6. For comparison, the monthly sunspot numbers are plotted for solar cycle 23 (1994- 2008). The expected curve for the solar cycle 25 in Figure 6 is drawn as the average of the solar cycles 23 and 24. The combination provides approximately the required expected amplitude for the solar cycle 25 .

First, the solar cycles 23 and 24 are scaled to have a period of 1.0. The data are interpolated to provide sufficient data points. Their amplitudes are added and divided by half to produce a new combined curve. The combined curve is scaled up in time to have the correct start time and the time for the expected maximum 13-month smoothed sunspot number $R_{25}$ to occur.

As shown in Figure 5 and the averages calculated above, a first major peak in the daily average of the sunspot numbers would likely occur in Fall 2022, while the 13-month smoothed sunspot numbers would peak about two years later, in early 2025.

Relating closely to the solar cycles, a phenomenon called magnetic poles reversal has been observed [20]. Presented below is the numerical calculation made for solar cycles 20 and 21 .

As described in the literature, an electromagnetic torque acts on a magnetic dipole in a rotating body of an angular velocity $\Omega$. The Maxwell differential equations involved in the electromagnetic field have been solved under different conditions [21-24]. The spin-down torque, $\tau_{z}$, has been observed, though there are still other unknown sources that have caused it to deviate from the theoretical value of 3 (e.g. $\mathrm{n}=2.91 \pm 0.05$ for Pulsar J1119-6127) [25]. In a Cartesian coordinate xyz system, the angular momentum $\mathrm{L}$ is directed along the $\mathrm{z}$-axis, the magnetic moment $\mathrm{M}$, which is lying in the yz plane, is inclined at a polar angle $\theta$ to the angular momentum L. The electromagnetic torque involved in the magnetic poles reversal is $\tau_{\mathrm{y}}[24,26]$, as shown in Equation (2),

$$
\tau_{\mathrm{y}}=\frac{2}{3} \frac{M^{2}}{c^{3}} \Omega^{3} \sin \theta \cos \theta
$$

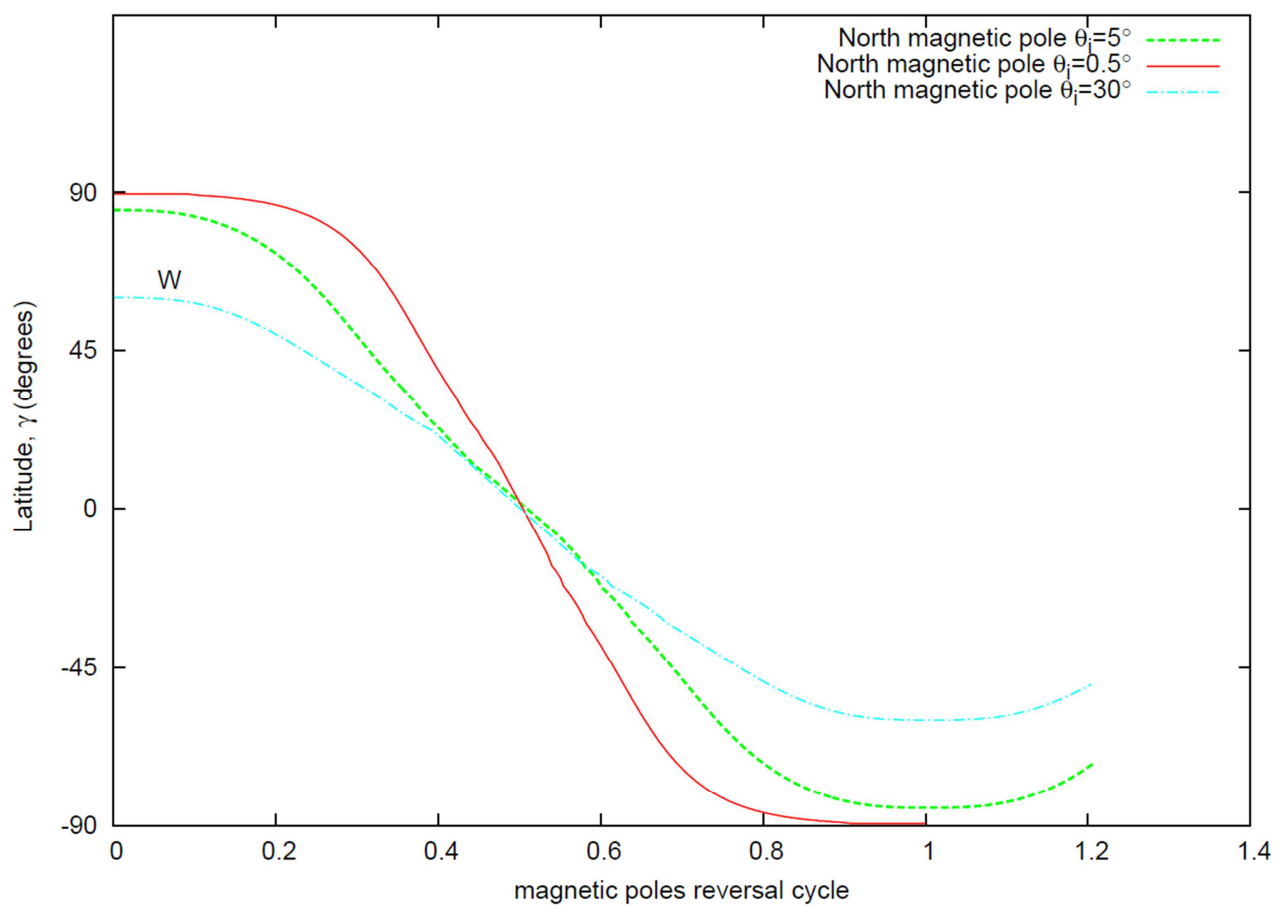

Figure 7. Numerical calculations for different initial angles of rotation in the magnetic poles reversal.

The rotation of a magnetic dipole having a polar angle $\theta$ for three initially different starting angles $\theta_{\mathrm{i}}=0.5^{\circ}, 5^{\circ}$ and $30^{\circ}$, is plotted as shown in Figure 7 . It has been known that two opposing magnetic poles occupy the north and south polar caps at solar minimum $[20,27]$. For the north magnetic pole and the south magnetic pole, the latitude during solar minimum is, $\gamma \sim \pm 90^{\circ}$, the value of the north polar angle is, $\theta$ $\sim 0^{\circ}$. A curve "W" with a starting polar angle $\theta_{\mathrm{i}}=30^{\circ}$ is shown in Figure 7. The curve "W" is inconsistent with the observed trajectory of the Sun's north magnetic pole poles, as it would have implied that $\gamma= \pm 60^{\circ}$ near solar minimum. It is concluded that the Sun's magnetic dipole has started with an initially small polar angle $<10^{\circ}$ from its rotation axis.

Figure 8 is a plot of the polar magnetic field strength recorded for the solar cycles 21 and 22 [28, 29]. The Sun's polar field strength is measured daily in the Wilcox Solar Observatory (WSO). The data in Figure 8 are reproduced from WSO (http://wso.stanford.edu/gifs/Polar.gif). 


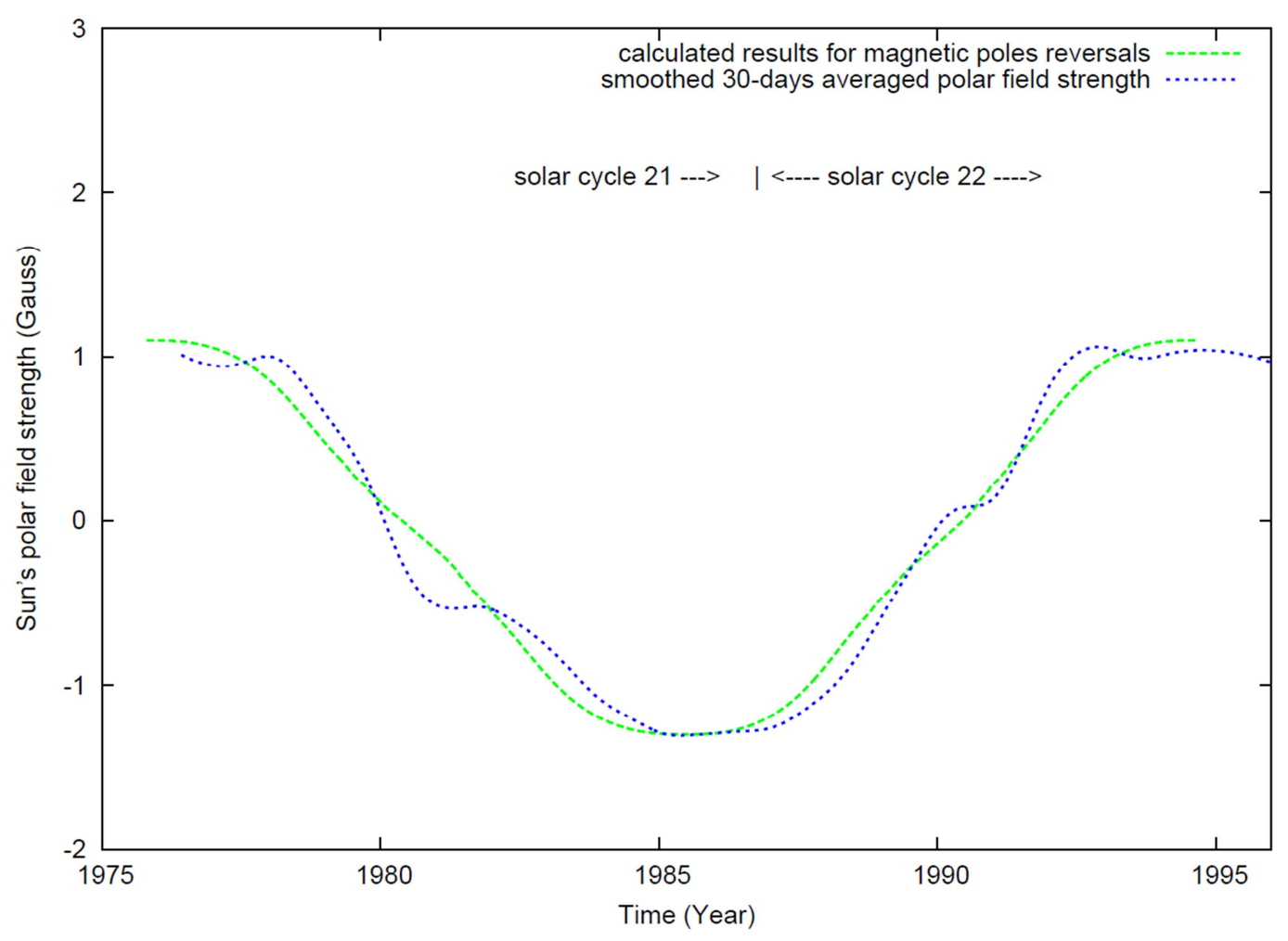

Figure 8. Polar magnetic field strength for solar cycles 21 and 22 (data reproduced from Wilcox Solar Observatory).

The results from the numerical calculation using Equation (2) are also shown in Figure 8. The starting polar angle is $\theta_{\mathrm{i}}$ $=5^{\circ}$. In the calculation, the period of the solar cycle 22 (1986.9-1996.8) is scaled by a factor of 0.9 , in comparison with the solar cycle 21 (1976.3-1986.9). The solar cycle 22 has a shorter period than the solar cycle 21. In Figure 8, it is shown that the two calculated magnetic poles reversals for solar cycles 21 and 22 are very close to the smoothed 30days averaged polar magnetic field strength given by the Wilcox Solar Observatory.

\section{Discussion}

The functional dependence of the magnetic poles reversal on the variable sine, which is a sinusoidal function as indicated in Equation (2), is clearly shown in Figure 8. The Equation (2), which is derived from the Maxwell's electromagnetic equations, applies when there is a rotating magnetic moment. The numerical coefficients in Equation (2) may be different under different boundary conditions. The functional dependency on the variables is usually valid, independent of the mechanism that generates the magnetic field. The introduction of the magnetic moment is to simplify the calculation to yield a bigger picture on the consequences from a rotating magnetic moment, irrespective of the details embedded in the magnetic sources, even if they are made from many individual sources.

A simplified method for processing geomagnetic activity data is presented above. Further improvement would be possible when many other factors are taken into consideration.

\section{Conclusion}

As shown above, it is useful to separate the extra contribution made by the coronal mass ejections from the geomagnetic activity. The geomagnetic data, collected so far, have indicated a new solar cycle with an activity which is not very much stronger than the current fading solar cycle 24 , thus joining one of the weak series of the sunspots activity in modern history.

\section{Acknowledgements}

The author would like to wholeheartedly thank Dr. Dean W. Pesnell of NASA's Goddard Space Flight Center for his critical review on the major portion of the manuscript. His invaluable suggestion and guidance have drastically improved the manuscript. The author would also like to express gratitude and deep appreciation to Professor Miriam Forman, Professor Paul Grannis, Professor Bob McCarthy and Professor Michael Rijssenbeek for their advice and help on the manuscript. The results presented in this paper rely on geomagnetic indices from ISGI Collaborating Institutes and magnetic observatories. We thank the involved national institutes, the INTERMAGNET network and ISGI (isgi.unistra.fr). We also thank the Wilcox Solar Observatory for the data on the polar field strength. The sunspots data were obtained from the WDC-SILSO, Royal Observatory of Belgium, Brussels. The websites used are: http://isgi.unistra.fr/ and http://www.sidc.be/silso/datafiles 


\section{References}

[1] Kappenman, J. G., L. J. Zanetti, and W. A. Radasky, Geomagnetic storm forecasts and the power industry. Eos Trans. AGU 78, 37 (1997).

[2] Shepherd Simon J., Zharkov Sergei I., Zharkova Valentina V. Prediction of solar activity from solar background magnetic field variations in cycles 21-23. ApJ. 795, 46 (2014).

[3] Gkana, A., Zachilas, L. Re-evaluation of predictive models in light of new data: Sunspot number version 2.0. Solar Phys. 291, 2457 (2016).

[4] Pesnell, W. D. Predictions of Solar Cycle 24: How are we doing? Space Weather 14 (1), 10-21 (2016).

[5] Javaraiah J. Long-term variations in the north-south asymmetry of solar activity and solar cycle prediction, III: Prediction for the amplitude of solar cycle 25. New Astron. 34, 54-64 (2015).

[6] Miao Juan, Gong J., Li Z., Ren T. The prediction of maximum amplitude of solar cycle 25. Scientia Sinica Physica, Mechanica \& Astronomica, 45, 099601 (2015).

[7] Helal, H. R., Galal, A. An early prediction of the maximum amplitude of the solar cycle 25. J. Adv. Res. 4 (3), 275-278, (2013).

[8] Yoshida, A., Difference between even- and odd-numbered cycles in the predictability of solar activity and prediction of the amplitude of cycle 25. Ann. Geophys. 32, 1035-1042, (2014).

[9] Lampropoulos G., Mavromichalaki H., Tritakis V. Possible Estimation of the Solar Cycle Characteristic Parameters by the $10.7 \mathrm{~cm}$ Solar Radio Flux. Solar Phys. 291 989-1002 (2016).

[10] Pesnell, W. D. and Schatten K. H. An Early Prediction of the Amplitude of Solar Cycle 25. Solar Phys. 293, 112 (2018).

[11] Pesnell, W. D. Predicting solar cycle 24 using a geomagnetic precursor pair. Solar Phys. 289, 2317-2331 (2014).

[12] Bhatt, Nipa J., Jain, Rajmal and Aggarwal, M. Predicting maximum sunspot number in solar cycle 24. Journal of Astrophysics and Astronomy 30, 71 (2009).

[13] $\mathrm{Du}$ Z. L. The correlation between solar and geomagnetic activity. Ann. Geophys. 29, 1005- 1018, (2011).

[14] Ng, K. K. Prediction Methods in Solar Sunspots Cycles. Scientific Reports 6, 21028 (2016).
[15] Ohl A. I. Wolf's number prediction for the maximum of the cycle 20. Soln. Dannye. 12, 84 (1966).

[16] Thompson, R. J. A technique for predicting the amplitude of the solar cycle. Solar Physics. 148, 383-388 (1993).

[17] Feynman, J. Geomagnetic and solar wind cycles, 1900-1975. J. Geophys. Res. 87, 6153-6162 (1982).

[18] Lockwood M., Stamper R., and Wild M. N., A doubling of the Sun's coronal magnetic field during the past 100 years. Nature 399, 437-439 (1999).

[19] Winter L. M., Balasubramaniam K. S. Estimate of solar maximum using the 1-8 A geostationary operational environmental satellites x-ray measurements. ApJ. L., 793, L45, (2014).

[20] Babcock, H. D., The Sun's polar magnetic field. ApJ, 130, 364, (1959).

[21] Deutsch, A. J. The electromagnetic field of an idealized star in rigid rotation in vacuo. Annales D'Astrophysique. 18, 1 (1955).

[22] Melatos A. Radiative precession of an isolated neutron star. MNRAS. 313, 217 (2000).

[23] Zanazzi, J. J. \& Lai D. Electromagnetic torques, precession and evolution of magnetic inclination of pulsars. MNRAS. 451, 1, 695-704 (2015).

[24] Good, M. L. \& Ng, K. K. Electromagnetic torques, secular alignment, and spin-down of neutron stars. ApJ. 299, 706 (1985).

[25] Livingstone, M. A., Kaspi, V. M., Gavriil, F. P., Manchester, R. N., Gotthelf, E. V., Kuiper, L., New Phase-coherent Measurements of Pulsar Braking Indices, Astrophysics and Space Science 308, 1-4, (2007).

[26] Ng, K. K. Relativistic correction to the movement of magnetic poles. ApJ. 714, 675-679 (2010).

[27] Smith et al. The Sun and heliosphere at solar maximum. Science. 302, 1165 (2003).

[28] Hoeksema, J. T. The large scale structure of the heliospheric current sheet during the ULYSSES epoch. Space Sci. Reviews 72, 137-148 (1995).

[29] Svalgaard, L., Duvall, T. L. Jr., Scherrer, P. H. The strength of the Sun's polar fields. Solar Physics 58, Issue 2, 225-239 (1978). 